\title{
Vibration Characteristics of Roundabout Swing of HAWT Wind Wheel
}

\author{
Jian-long Ma, ${ }^{1,2}$ Pei-lin Li, ${ }^{1}$ Jian-wen Wang, ${ }^{1,2}$ Liu Yang, ${ }^{1}$ and Yan-qi Zhang ${ }^{1}$ \\ ${ }^{1}$ School of Energy and Power Engineering, Inner Mongolia University of Technology, Hohhot 010051, China \\ ${ }^{2}$ Ministry of Education Key Laboratory of Wind Energy and Solar Energy Utilization Technology, Hohhot 010051, China
}

Correspondence should be addressed to Jian-long Ma; ma_jianlong@yeah.net

Received 13 March 2016; Revised 12 June 2016; Accepted 22 June 2016

Academic Editor: Vadim V. Silberschmidt

Copyright (c) 2016 Jian-long Ma et al. This is an open access article distributed under the Creative Commons Attribution License, which permits unrestricted use, distribution, and reproduction in any medium, provided the original work is properly cited.

Modal testing was used to show that the roundabout swing was a natural vibration mode of the wind wheel of a horizontal-axis wind turbine (HAWT). During the vibration, the blade root was simultaneously subjected to bending and rotary shear stresses. A method for indirect testing and determination of the dynamic frequencies of the typical vibrations of the wind wheel was developed, based on the frequency-holding characteristic of each subsignal during the transmission of the multiple mixed-vibration signals. The developed method enabled simple and accurate acquisition of the dynamic frequencies without destruction of the flow and structural fields. The dynamic vibration stress of the roundabout swing was found to be significantly stronger than those of the firstand second-order flexural vibrations of the blades. By a combination of numerical simulations and tests, it was determined that the pneumatic circumferential force was the primary determinant of the roundabout swing vibration frequencies, the relationship being quadratic. The roundabout swing vibration potentially offers new explanations and analytical pathways regarding the behavior of horizontal-axis wind turbines, which have been found to be frequently involved in fatigue-damage accidents within periods shorter than their design lives.

\section{Introduction}

The wind wheel is a key power component and the primary excitation source of a wind turbine. Its natural vibration characteristics are thus considered to be the basic considerations in the safe structural design of a wind turbine [1]. The natural vibration parameters of a wind wheel vary with the working conditions, and this has gradually attracted serious attention and became a hot topic with respect to current active developments of large- and small-scale multipurpose wind turbines [2-5]. However, the progress of relevant studies has been relatively slow due to the lagging development of new test and numerical analysis methods.

Researchers have tended to focus on the first- and secondorder vibration modes of the wind wheel and the accurate determination of the vibration parameters $[6,7]$, ignoring the first-order following low-frequency vibrations. There have been even fewer studies on the effect of the regularity of the various vibration stresses on the structural safety and the main control features of the various vibration modes.
This has directly resulted in the inability of scholars to proffer a complete theoretical explanation and solution to the frequent fatigue-damage accidents involving wind turbines within periods shorter than their design lives. It has also led to the structural failure of many innovative wind wheels despite their excellent aerodynamic performance. The main reasons for this are as follows:

(1) Traditional idea of inertia obtained from previous studies: researchers have held the idea that the bending vibrations caused by the aerodynamic force were the most important aspect of the vibration characteristics of a wind wheel [8-10]. This has led to the relative neglect of the other types of vibration.

(2) Technical challenges of testing: wind wheels are rotating machineries, and those used in HAWTs particularly often have higher design speeds for higher power coefficients. This makes it difficult to install sensors directly on the blade surface [11-14]. Besides, the results of direct testing are less accurate because 


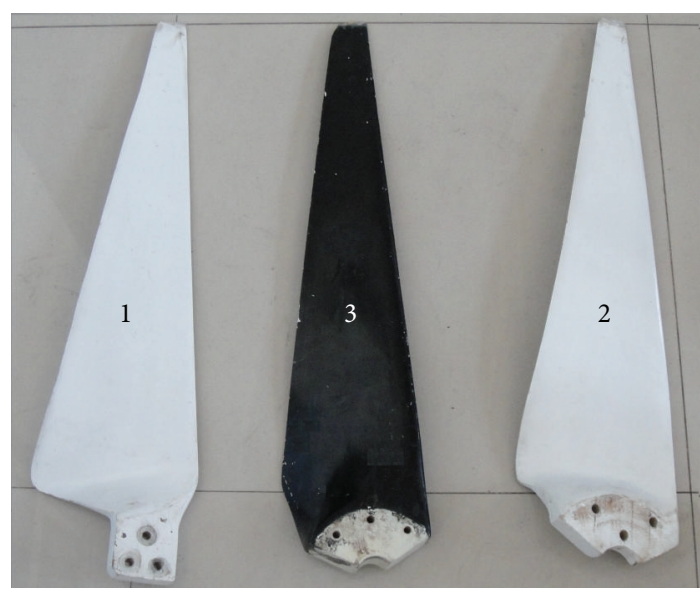

FIGURE 1: Three considered blade types.

the sensors severely distort the original airfoil structure of the blade and its mass distribution [15-17].

(3) Numerical simulation challenges: the numerical determination of the dynamic vibration characteristics of a wind wheel is a typical fluid-structure interaction problem, and studies along this line have been significantly restricted by the hysteretic development of fluid-structure interaction theories and inadequate computational methods [18-20].

In view of the foregoing, the present study employed modal testing of the low-frequency vibration characteristics of $1.4 \mathrm{~m}$ diameter wind wheel to obtain valuable information. The aim was to develop an indirect testing method for determining the dynamic frequencies of a wind wheel through a combination of spectral analysis and modal testing, with the purpose of determining the vibration stresses in various vibration modes using the classical Newtonian mechanics formula. This is expected to enable analysis of the different vibration stresses in the static and dynamic states, as well as their significance. We also used a combination of numerical calculations and tests to analyze the response characteristics of the low-frequency vibration of the wind wheel with respect to the aerodynamic and centrifugal forces.

\section{Determination and Analysis of Characteristics of Roundabout Swing}

2.1. Blade Types. Three different types of blades were considered for the investigated $1.4 \mathrm{~m}$ diameter wind wheel. All the blades were made from wood and their roots were connected by the flange method using double plywood. Figure 1 shows the three blade types.

Blade number 1 had an innovative airfoil recently developed by the authors. Blade number 2 had the same airfoil but a different connection mode, which was used to examine whether the roundabout swing was due to the connection mode. Blade number 3 had NACA4415 airfoil and was larger than blade number 1 . It was used to examine whether the roundabout swing was due to the airfoil.
TABLE 1: Typical second-order following vibration frequencies.

\begin{tabular}{lcccccc}
\hline $\begin{array}{l}\text { Blade } \\
\text { number }\end{array}$ & $f_{\mathrm{AO}}(\mathrm{Hz})$ & $f_{\mathrm{RS}}(\mathrm{Hz})$ & $f_{1 \mathrm{~A}}(\mathrm{~Hz})$ & $f_{1 \mathrm{~S}}(\mathrm{~Hz})$ & $f_{2 \mathrm{~A}}(\mathrm{~Hz})$ & $f_{2 \mathrm{~S}}(\mathrm{~Hz})$ \\
\hline 1 & 9.95 & 17.10 & 23.40 & 24.90 & 72.70 & 77.20 \\
2 & 9.73 & 17.90 & 19.80 & 23.30 & 87.70 & 90.10 \\
3 & 8.00 & 15.00 & 21.60 & 24.50 & 81.80 & 87.90 \\
\hline
\end{tabular}

2.2. Test System and Method. The test system was PULSE 19.1 system developed by the Danish B\&K Company. The test was conducted on a dedicated test bench installed in front of the opening of B1/K2 low-speed wind tunnel. The test method involved transient excitation, one-point driving, and multipoint response. The test system and setup are shown in Figure 2.

2.3. Data Processing and Analysis. The test data was processed by the $\mathrm{ME}^{\prime}$ scope 5.1 software. The distribution of the measurement points on the wind wheel is shown in Figure 3.

As an example, the acquired natural frequencies of blade number 1 are shown in Figure 4.

The typical second-order following vibration modes and frequencies of the three wind wheel blades are presented in Table 1. The vibration stress of an axial wobble (the roundabout swing) is expressed as $F_{\mathrm{AO}}\left(F_{\mathrm{RS}}\right)$, while the stress of the first-order antisymmetric vibration (first-order symmetric vibration, second-order antisymmetric vibration, and second-order symmetric vibration) is expressed as $F_{1 \mathrm{~A}}\left(F_{1 \mathrm{~S}}\right.$, $\left.F_{2 \mathrm{~A}}, F_{2 \mathrm{~S}}\right)$. Similarly, the vibration frequencies are expressed sequentially as $f_{\mathrm{AO}}, f_{\mathrm{RS}}, f_{1 \mathrm{~A}}, f_{1 \mathrm{~S}}, f_{2 \mathrm{~A}}$, and $f_{2 \mathrm{~S}}$.

The following can be deduced from the test results in Figure 5:

(1) The roundabout swing is a natural vibration mode of a wind wheel. Considering the structural distinctions of the three blades, the roundabout swing is not caused by the blade connection method or the airfoil structure.

(2) The quality of the wind wheel was stable during the test. Based on the classical formula $F=m \cdot a$, where $m$ indicates the quality and $a$ indicates the acceleration, the magnitude of the wheel acceleration reflected that of the vibration stress. In other words, $F_{2 \mathrm{~A}}\left(F_{2 \mathrm{~S}}\right)>$ $F_{1 \mathrm{~A}}\left(F_{1 \mathrm{~S}}\right)>F_{\mathrm{RS}}>F_{\mathrm{AO}}$. This also largely applies to the other two types of vibration stresses.

Considering blade number 1 as an example, the roundabout swing vibration mode is shown in Figure 5.

The vibration process of the roundabout swing is illustrated by the nine contiguous dynamic screenshots in Figure 5. The immobile line surfaces are those of the static blades, while the curved ones are those of the vibrating blades in different positions.

The vibration frequency of the roundabout swing is lower than that of the first-order bending vibration. This is because the overall effectiveness of the wind turbine is significantly higher than that of a single blade. The vibration causes the roots of the blades to bear the stress, which comprises curved 


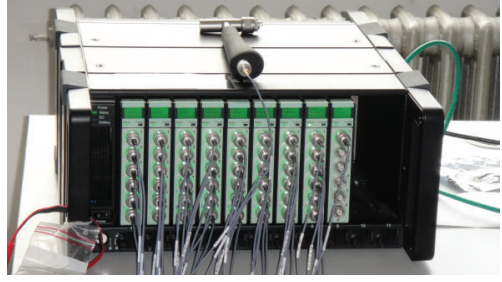

(a) PULSE 19.1 system

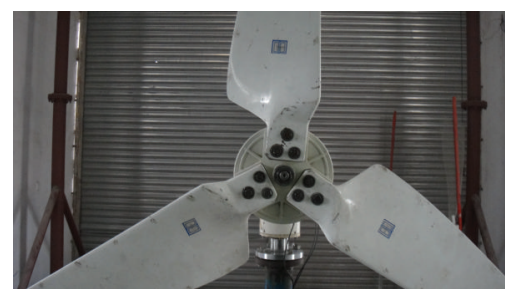

(b) Flange connection

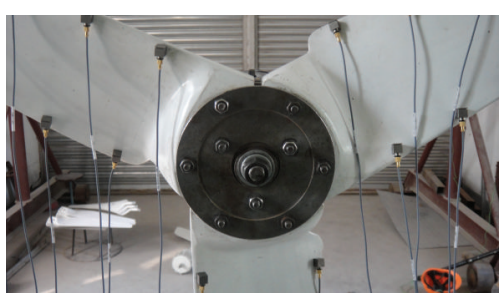

(c) Double plywood

Figure 2: Modal testing.

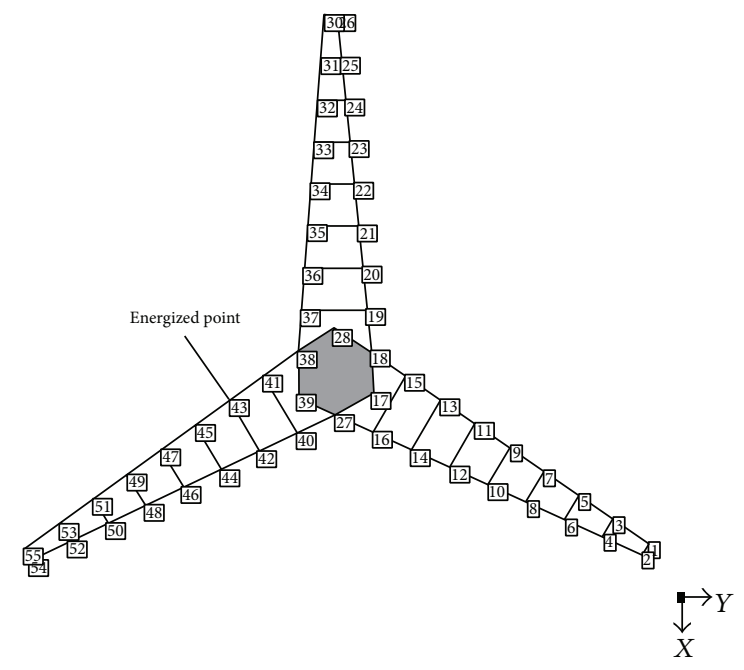

Figure 3: Distribution of measurement points on wind wheel.

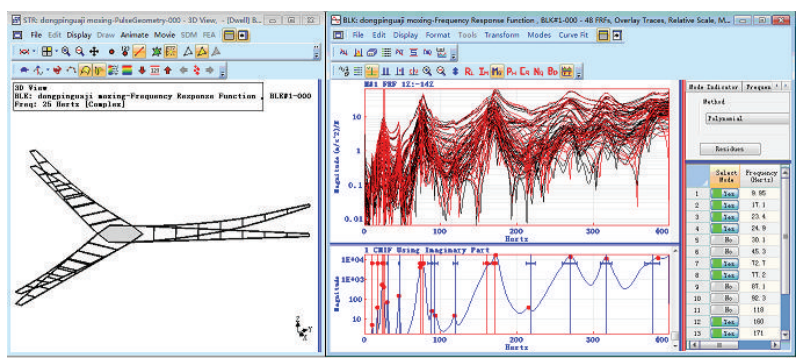

FIgURE 4: Vibration frequencies of blade number 1.

shear stress and rotary shear stress. This is because a single blade is similar to a cantilever beam with significant first- and second-order bending vibration performances. The entire wind wheel is thus characterized by whirling vibration owing to the three blades forming a circular frame. Considering that the roundabout swing is easily triggered over time, it hastens fatigue damage of the blades, thereby shortening their service life.

\section{Vibration Characteristics of Roundabout Swing under Operation Conditions}

During the rotation of a wind wheel, forced vibration is induced by aerodynamic, centrifugal, and gravitational forces. This external excitation increases the deformation and stiffness of the blade, resulting in the typical vibration frequencies of the wind wheel exceeding the natural vibration frequencies. Although the external excitation is different, it may affect the vibration frequency, amplitude, and phase change, but not the vibration mode. The natural modes and frequencies of the wind wheel can thus be initially determined by modal testing, based on which the dynamic vibration frequency can be obtained by spectral analysis.

3.1. Test Object, System, and Method. The test object and system were the same as those of the modal test. Considering that the installation of the acceleration sensors on the surfaces of the blades would significantly alter their shapes and distort the aerodynamic force and mass distribution, an indirect test and analysis method for determining the typical dynamic vibration frequencies of the wind wheel was developed. The developed method is based on the frequency-holding characteristic of each subsignal during the transmission of multiple mixed-vibration signals. Specifically, the acceleration signal from the accelerometer installed at the front end of the generator is received, and its spectral signature is then obtained by FFT. Finally, the dynamic frequencies are distinguished based on their mode shapes and spectral signatures.

Figures 6 and 7, respectively, show the test system for determining the dynamic frequencies and the test setup.

The vibration signals were received by four three-dimensional acceleration sensors. As shown in Figure 7, sensors numbers 1-4 were, respectively, installed at the top of the front end of the generator, at the front end of the generator sidepiece (the distance to the wind wheel is slightly larger than the size of sensor number 1), midway on the top of the generator, and at the lower end of the generator.

3.2. Reliability Analysis of Test Results and Test Method. Using blade number 1 as an example, we analyzed the axial vibration signals collected by the four sensors at a wind velocity of $10 \mathrm{~m} / \mathrm{s}$ and tip speed ratio of 6 . The vibration spectrums are shown in Figure 8.

An examination of Figure 8 showed that the dynamic spectrums obtained by sensors numbers 1 and 2 were very similar. Hence, the two spectrums were closely compared using a picture composite algorithm. It was found that the peak frequencies of the curves coincided, although those for sensor number 2 were slightly lower, which was because the distance from sensor number 2 to the wind wheel was slightly 


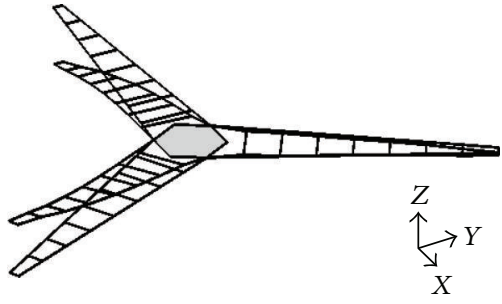

(a)

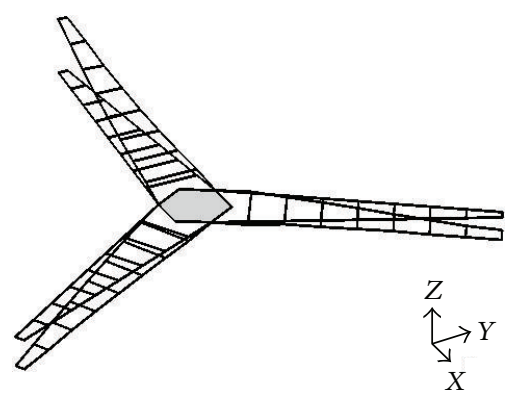

(d)

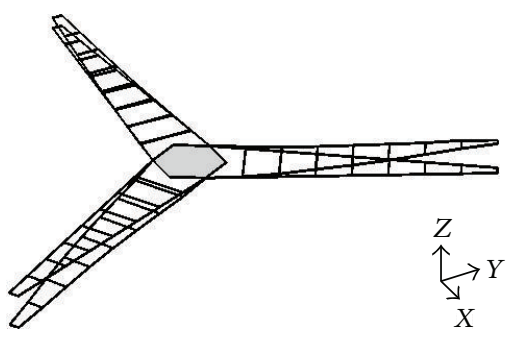

(g)

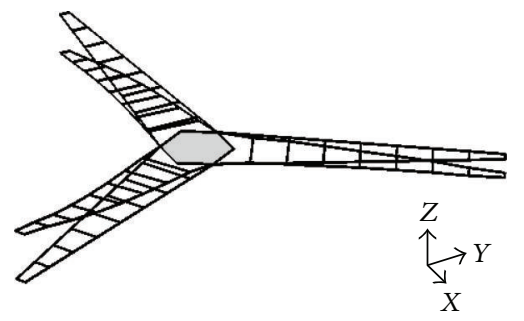

(b)

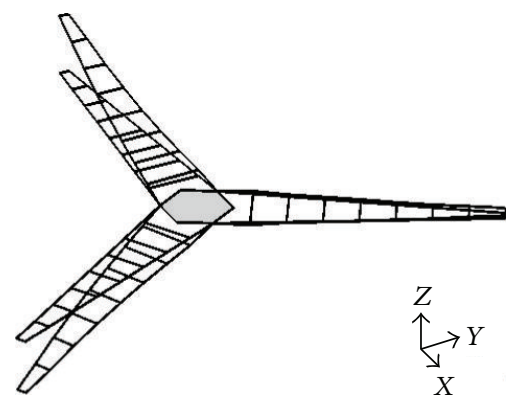

(e)

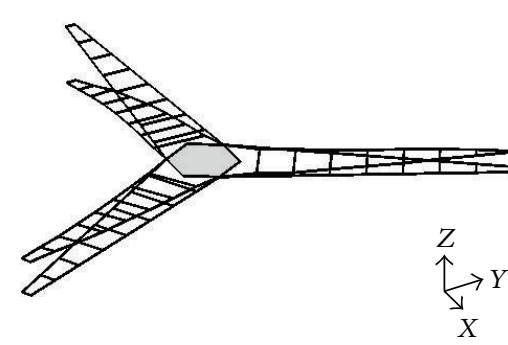

(h)

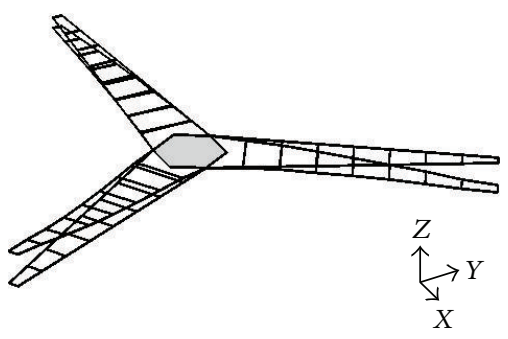

(c)

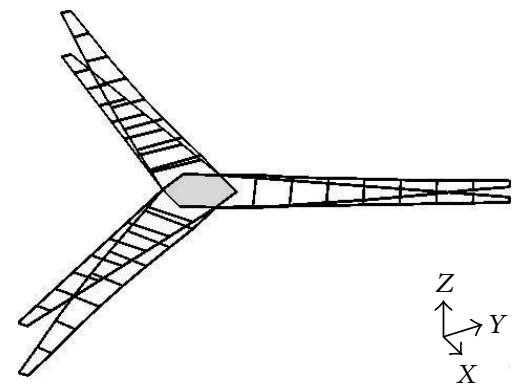

(f)

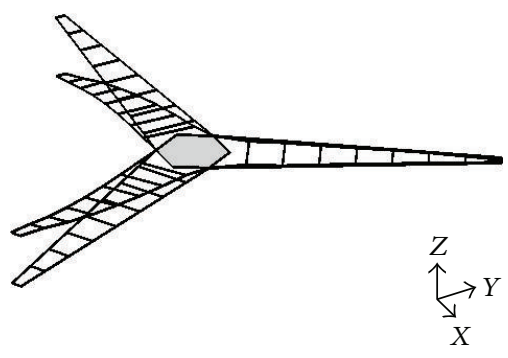

(i)

FIGURE 5: Vibration mode of roundabout swing.

longer than that of sensor number 1, resulting in a longer signal transmission path and slightly greater amplitude attenuation.

In the case of sensor number 3, which was farther from the wind wheel, the peak vibration spectrum gradually blurred with the reduction progressively increasing. This was due to the significant effect of the vibration of the generator excitation coil. The signals of sensor number 4 were even more significantly affected by the vibrations of the generator excitation coil and the tower.

The foregoing indicates that the dynamic frequency of the typical vibration mode of the wind wheel can be more ideally determined using the dynamic frequency curve of sensor number 1 . The determination of the dynamic frequency of the wind wheel is illustrated in Figure 9.

$f_{1 \mathrm{I}}$ is the vibration frequency of the first intermediate vibration, and $f_{2 \mathrm{I}}$ is the vibration frequency of the second intermediate vibration.

3.3. Analysis of Test Results. From Figure 9(b), it can be concluded that the second-order following vibration stresses during the rotation of the wind wheel were as follows: $F_{\mathrm{RS}}>$
$F_{1 \mathrm{~A}}\left(F_{1 \mathrm{~S}}\right)>F_{\mathrm{AO}}>F_{2 \mathrm{~A}}\left(F_{2 \mathrm{~S}}\right)$. This significantly differs from the indications of the modal test results.

The roundabout swing is primarily of concern under the operation conditions because its vibration stress is much higher than those of the other vibrations. The dynamic vibration frequencies of the roundabout swing vary with the operation conditions, especially the aerodynamic and centrifugal forces, and require intensive study because they are primary considerations in the structural safety design of a wind turbine.

\section{Effect of Aerodynamic and Centrifugal Forces on Vibration Frequencies of Roundabout Swing}

The following analysis was performed by numerical simulation considering its convenience, requiring only the loading of the aerodynamic and centrifugal forces.

4.1. Theoretical Model. Steady computing using the shear stress transport (SST) equation was employed, taking into consideration the effect of the turbulent shear stress. The import boundary was based on the inlet velocity, while 


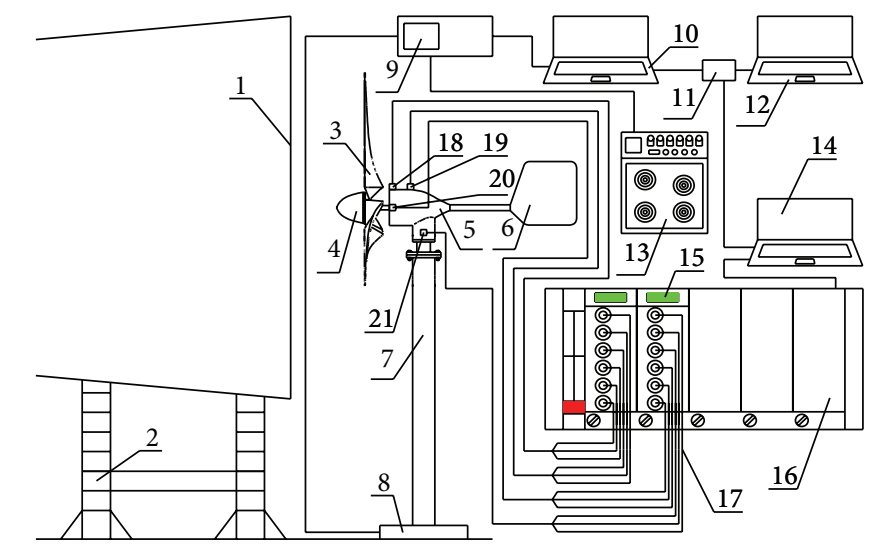

$\begin{array}{ll}\text { 1: wind tunnel } & \text { 11: synchronizer trigger } \\ \text { 2: bracket } & \text { 12: master computer } \\ \text { 3: blade } & \text { 13: constant-temperature load box } \\ \text { 4: air deflector } & \text { 14: acceleration signal acquisition } \\ \text { 5: electric generator } & \text { and control computer } \\ \text { 6: tail rudder } & \text { 15: data acquisition card } \\ \text { 7: tower } & \text { 16: data collection box } \\ \text { 8: foundation bed } & 17: \text { data wire } \\ \text { 9: generator output signal collector } & 18-21: \text { accelerometers } \\ \text { 10: generator output signal } & \\ \quad \text { acquisition and control computer } & \end{array}$

Figure 6: Dynamic frequency test system.

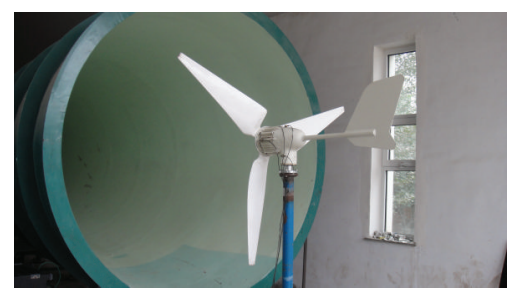

(a) Test bench

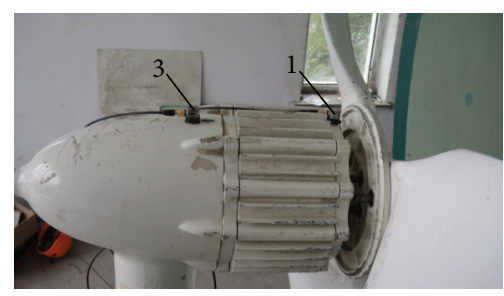

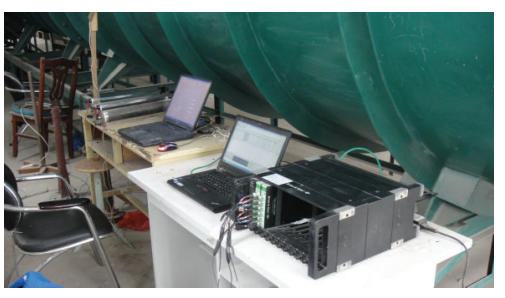

(b) Data acquisition

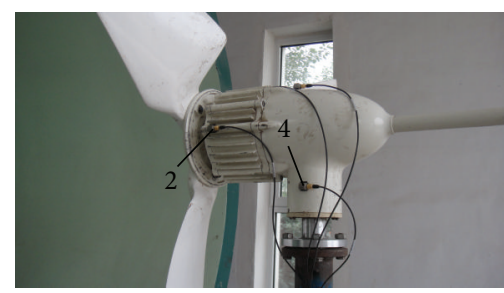

(c) Arrangement of sensors

Figure 7: Test setup.

the outlet boundary utilized pressure export, with a relative pressure of 0 . The energy, momentum, continuity, and SST equations were used to obtain the coupling solution. The calculation was performed using the unidirectional fluidstructure interaction computation method. The precalculated flow field data for different operation conditions were imputed, and the centrifugal force was then added to solve the structural dynamics equations and obtain the dynamic vibration parameters of the wind wheel, such as the vibration modes and vibration frequencies.

4.2. Generation of Computational Domain. Using blade number 1 as an example, the overall computational domain was generated to include the B1/K2 experimental wind tunnel, as shown in Figures 10 and 11. 


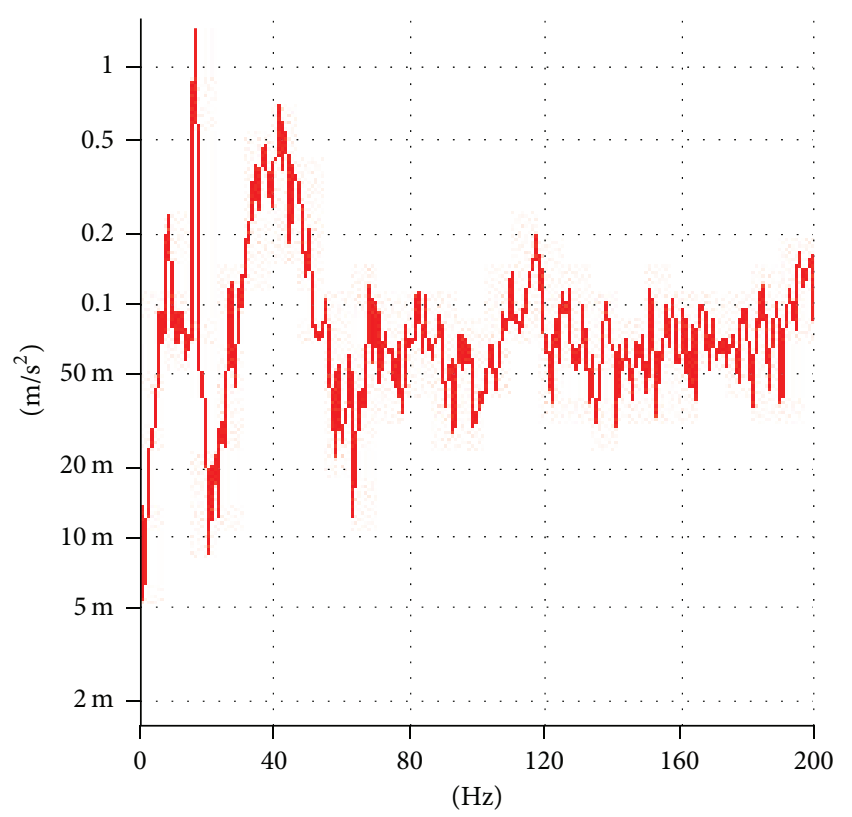

(a) Sensor number 1

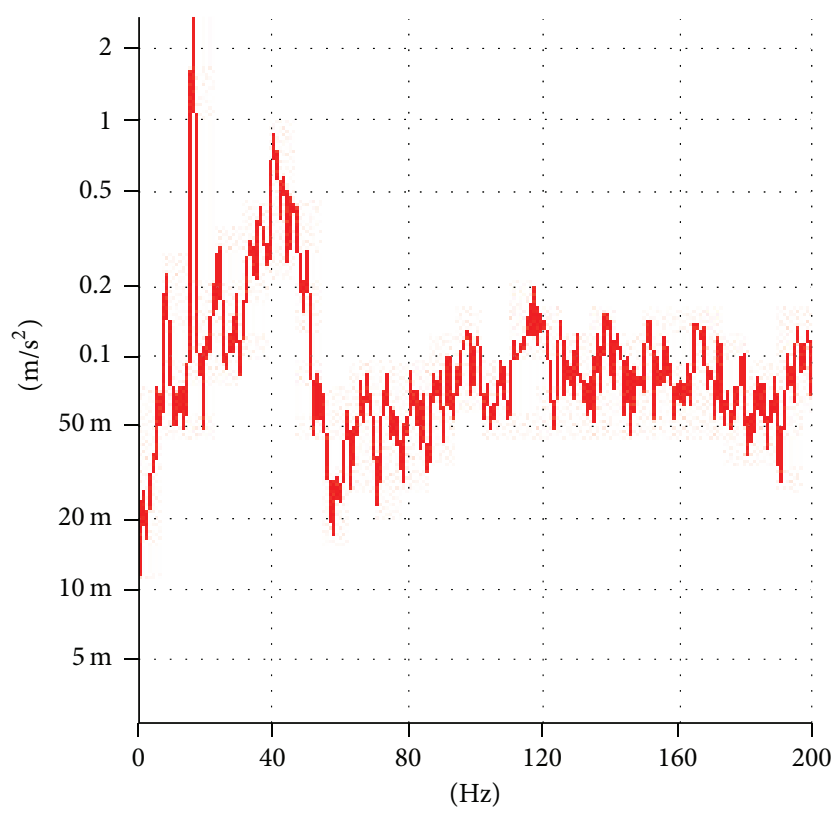

(c) Sensor number 3

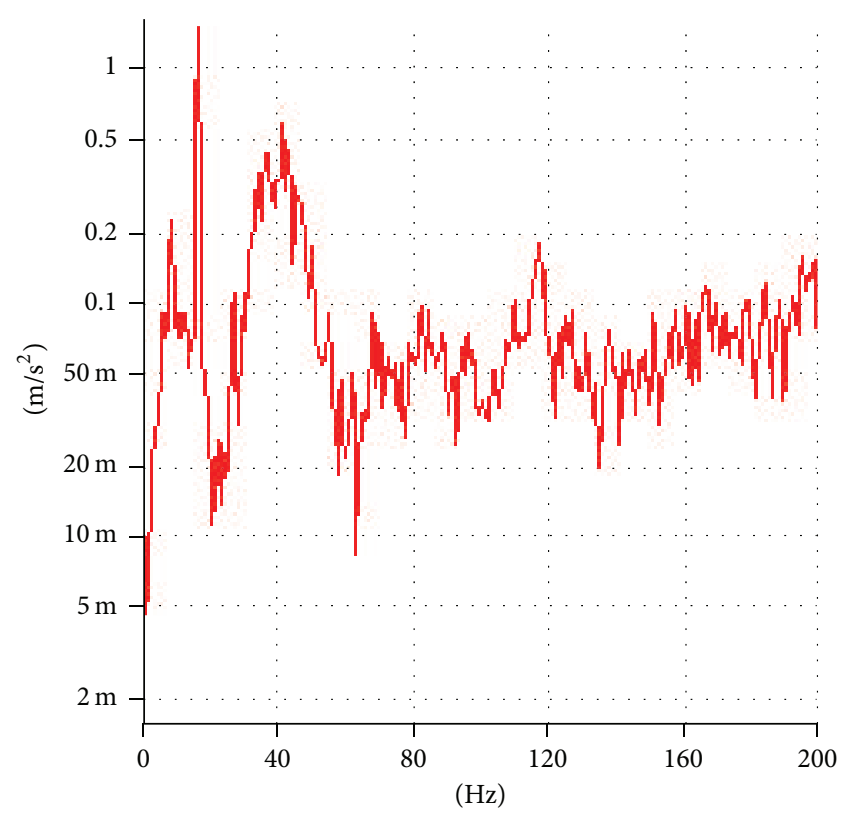

(b) Sensor number 2

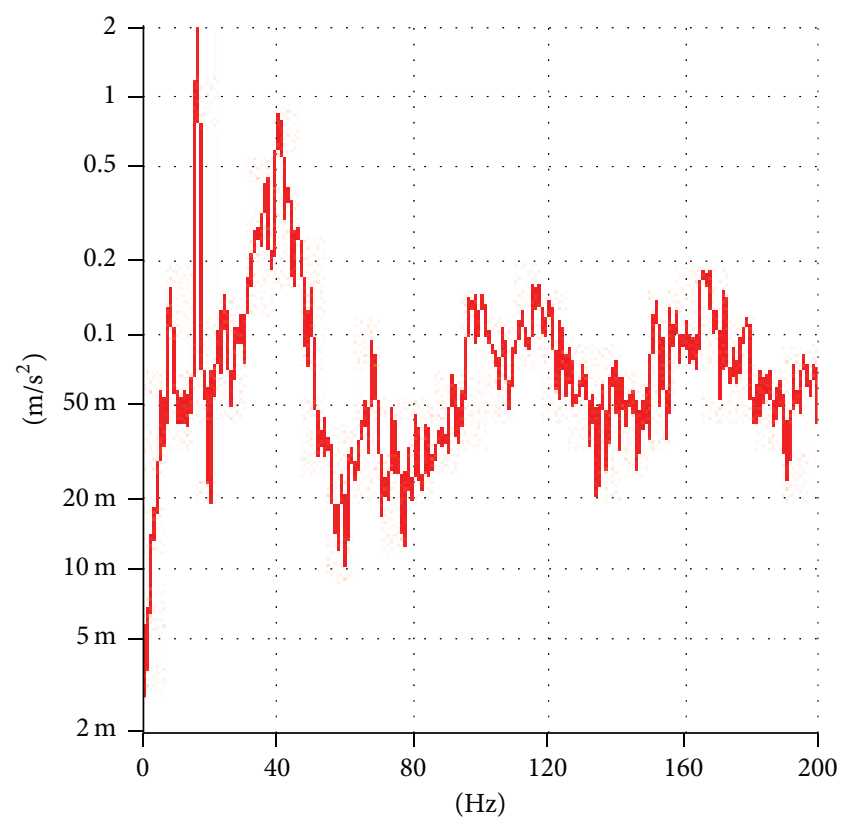

(d) Sensor number 4

FIGURE 8: Dynamic frequency spectrums.

4.3. Meshing. The computation domain was divided into two parts, namely, the rotation field and the static domain. The rotation field enclosed the blades, the rotation of which was simulated by rotating the field. The rotation region of the wind wheel was generated by a sliding mesh, with a no-slip condition at the stationary wall. The rotation domain was generated by a hexahedral mesh, with the static domain comprising a tetrahedral mesh and a sliding grid adopted between the two domains. The data transfer was done by interface technology.
To better capture the flow field information, a body-fitted grid was applied in the vicinity of the blade surface. The grid expansion technique was implemented in the static domain to partition the mesh layer. The full application of different available grid partitioning technologies generally enables efficient utilization of computing resources.

To properly assess the effectiveness of the mesh generation technique, the grid scale in Figure 12 has been enlarged; the actual grid was more refined than shown in the figure. 


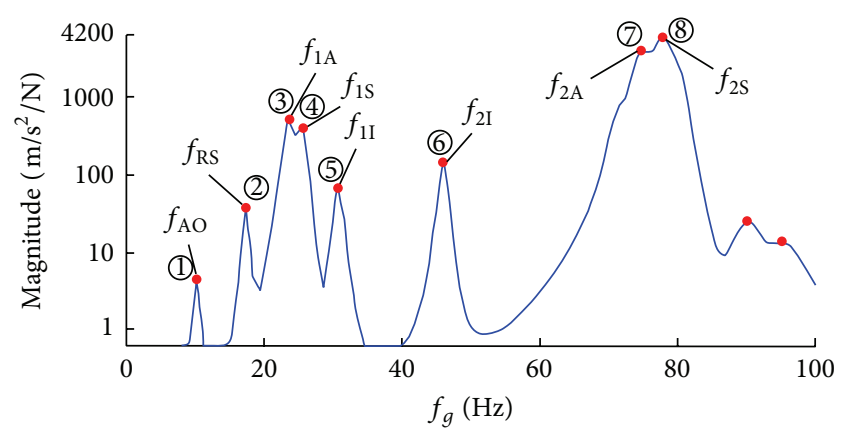

(a) Modal test spectrum

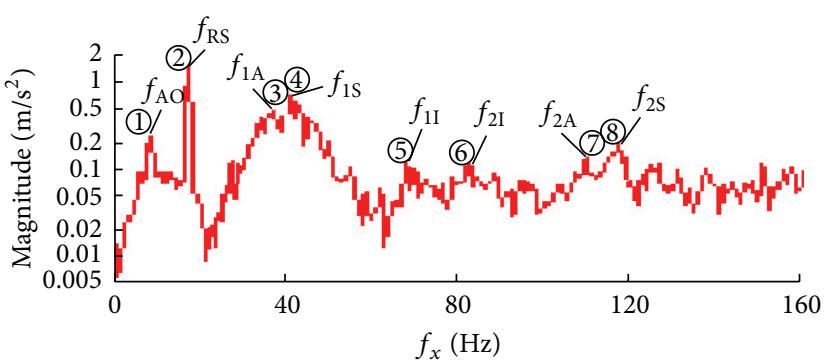

(b) Dynamic test spectrum

FIgURE 9: Determination of dynamic frequency of wind wheel.

TABLE 2: Dynamic frequencies of typical vibrations.

\begin{tabular}{lcccccc}
\hline Category & $f_{\mathrm{AM}}$ & $f_{\mathrm{DE}}$ & $f_{1 \mathrm{~A}}$ & $f_{1 \mathrm{~S}}$ & $f_{2 \mathrm{~A}}$ & $f_{2 \mathrm{~S}}$ \\
\hline Experimental value $(\mathrm{Hz})$ & 10 & 17.5 & 38 & 40.5 & 109 & 115.5 \\
Calculated value $(\mathrm{Hz})$ & 9.8 & 17.3 & 36.7 & 42.2 & 107.4 & 119.7 \\
Fractional error $(\%)$ & -2.0 & -1.1 & -3.4 & 4.2 & -1.5 & 3.6 \\
\hline
\end{tabular}

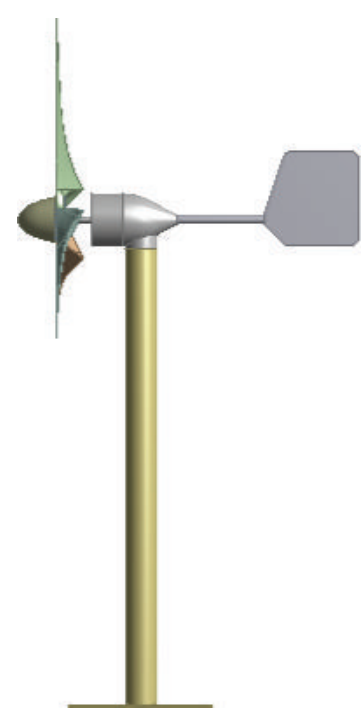

Figure 10: Wind turbine.

4.4. Computation Results and Analysis. Using blade number 1 as an example, the second-order following vibration modes are shown in Figure 13, where the black line in each panel indicates the position of the wind wheel in the static state. Dynamic frequencies of typical vibrations are shown in Table 2 at a wind velocity of $10 \mathrm{~m} / \mathrm{s}$ and tip speed ratio of 6 , where the aerodynamic force and the centrifugal force are loaded synchronously.

As shown in Figure 13, the axial oscillations were due to the elasticity of the tower. The other vibrations were natural vibrations, unrelated to the structural properties of the generator and tower.

To analyze the effects of the aerodynamic and centrifugal forces on the vibrating frequencies of the roundabout swing,

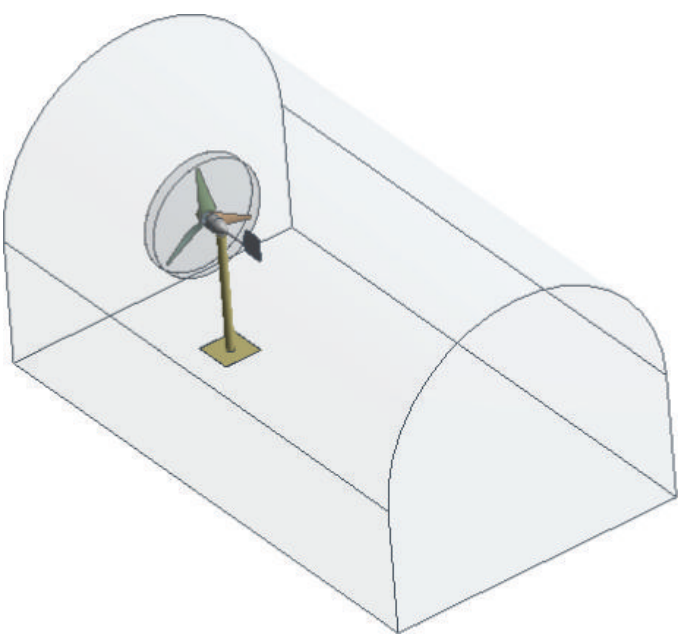

FIgURE 11: Computational domain.

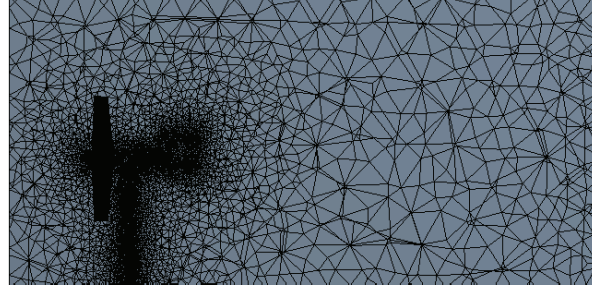

(a)

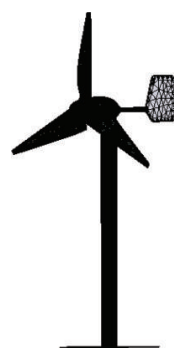

(b)
FIgURE 12: Meshing.

the two following example numerical calculations were performed:

Example 1. The tip speed of the wind wheel was $40 \mathrm{~m} / \mathrm{s}$; the wind speed was varied within $5-10 \mathrm{~m} / \mathrm{s}$. Two cases involving the application of only the aerodynamic force and the simultaneous application of the aerodynamic and centrifugal forces were, respectively, considered. The results are shown in Figure 14. 


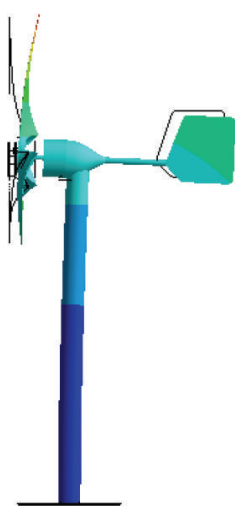

(a)

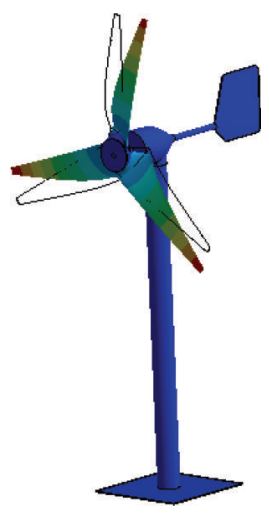

(b)

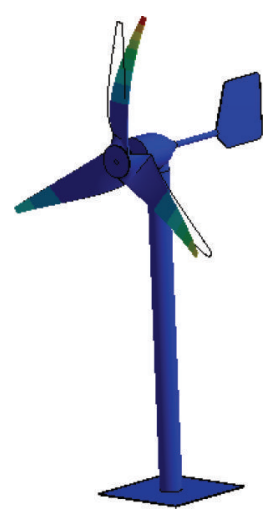

(c)

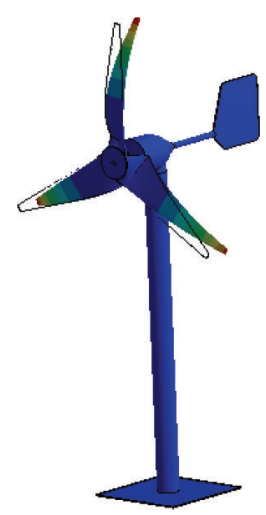

(d)

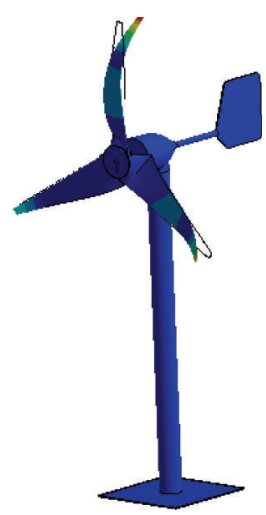

(e)

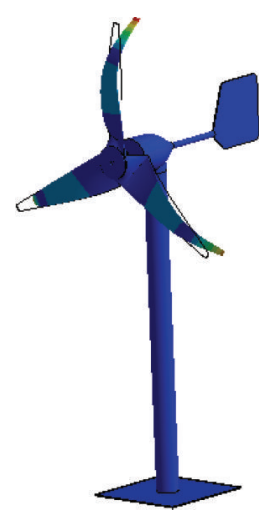

(f)

FIGURE 13: Determined vibration modes: (a) axial movement, (b) roundabout swing, (c) first-order antisymmetric vibration, (d) first-order symmetric vibration, (e) second-order antisymmetric vibration, and (f) second-order symmetric vibration.

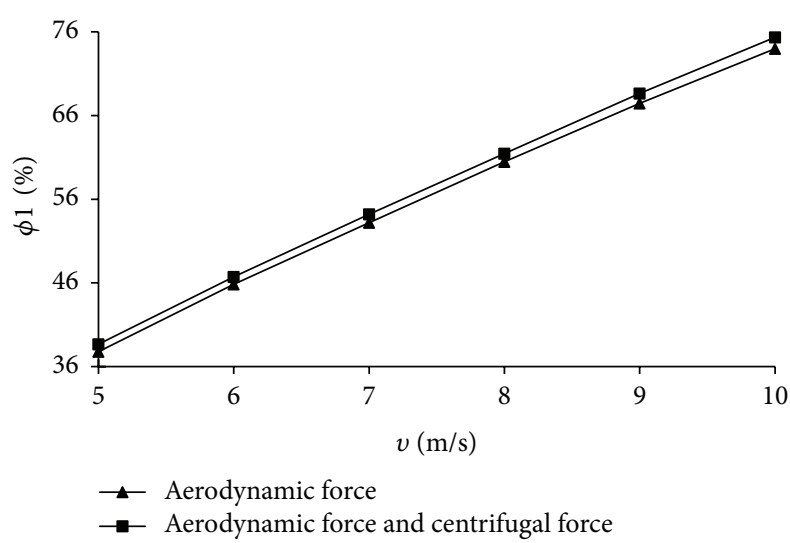

FIGURE 14: Variations of vibration frequencies of roundabout swing with working conditions defined by given tip speed and varying wind velocity.

Example 2. The wind velocity was $8 \mathrm{~m} / \mathrm{s}$, and the tip speed ratio of the wind wheel was varied within $4-8$. The two cases of example 1 were also considered here. The results are shown in Figure 15.

The vibration frequencies of the roundabout swing for the different wind speeds were expressed as $f_{i}(i=5,6,7,8$, $9,10 \mathrm{~m} / \mathrm{s}$ ), while the frequency increase was expressed as

$$
\phi 1=\frac{f_{i}-f_{5}}{f_{5}} \times 100 \%, \quad(i=5,6,7,8,9,10 \mathrm{~m} / \mathrm{s}) .
$$

The vibration frequencies of the roundabout swing for the different tip speed ratios were expressed as $f_{j}(j=$ $4,5,6,7,8)$, while the frequency increase was expressed as

$$
\phi 2=\frac{f_{j}-f_{4}}{f_{4}} \times 100 \%, \quad(j=4,5,6,7) .
$$

It can be determined from Figures 14 and 15 that the variation of the vibration frequencies of the roundabout swing with the aerodynamic force is of a highly sensitive quadratic

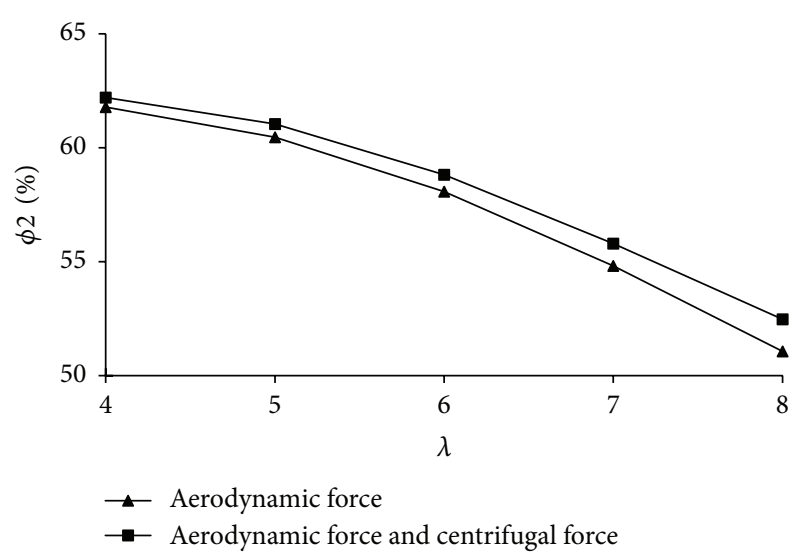

FIGURE 15: Variations of vibration frequencies of roundabout swing with working conditions defined by given wind velocity and varying tip speed ratio.

form. The centrifugal force, expressed in terms of the increase in the wind wheel speed, also increases the vibration frequencies, although the relationship is relatively insensitive.

For an in-depth analysis of the effect mechanism of the aerodynamic force on the vibration frequencies of the roundabout swing, an aerodynamic force that acted vertically on the surfaces of the blades was decomposed into an axial force in the axial direction of the wind wheel and a circumferential force in the circumferential direction of the wheel. The variations of the axial and circumferential forces with the working conditions are shown in Figures 16 and 17.

An increase or decrease in the vibration frequencies of a wind wheel is due to a stress-induced increase or decrease in the blade stiffness. Based on this theory, the variations of the pneumatic axial forces in Figures 16 and 17 can be observed to conflict with those of the vibration frequencies of the roundabout swing in Figures 14 and 15, although there is consistency in the case of the pneumatic circumferential forces. The pneumatic circumferential force is thus the primary determinant 


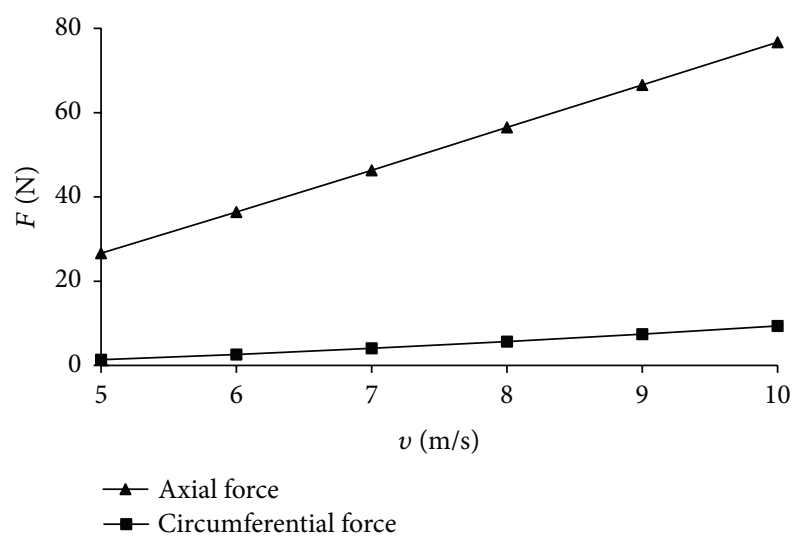

FIGURE 16: Variations of axial and circumferential forces with working conditions.

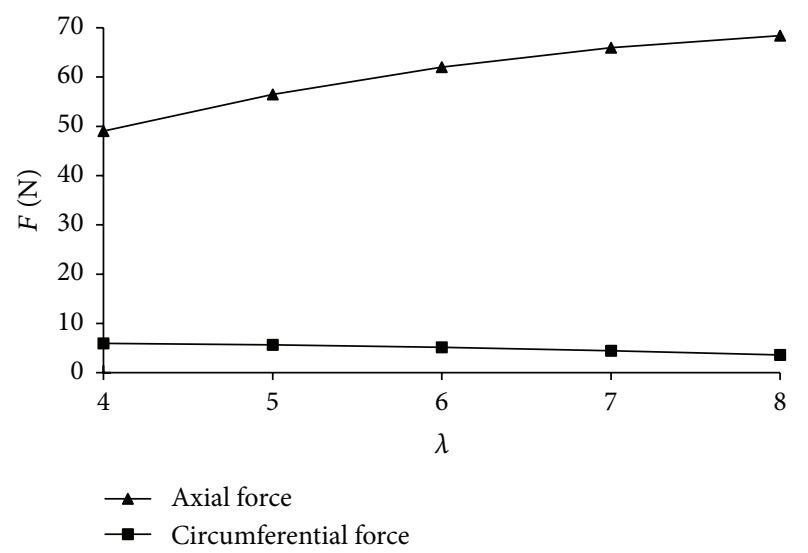

FIGURE 17: Variations of axial and circumferential forces with working conditions.

of the vibration frequencies, with the pneumatic axial force being a secondary factor.

To verify the above conclusion, the calculation data for the synchronous application of the aerodynamic and centrifugal forces was tested by comparison with experimental results. The relative error was determined to be less than $6 \%$.

\section{Conclusions}

(1) The roundabout swing is a natural vibration form of a wind wheel, with its vibration frequency less than the first-order bending vibration frequency and the vibration stress often significantly higher than the other present vibration stresses. This causes the blade roots to bear the compound stress, which comprises bending shear stress and rotary shear stress.

(2) The proposed indirect test method provides a solution to the technical difficulties associated with monitoring and distinguishing the dynamic frequencies of the typical vibrations of a wind wheel under operation conditions. The various vibration stresses in the static and dynamic tests of the present study were found to be characterized by $F_{\mathrm{RS}}>F_{1 \mathrm{~A}}\left(F_{1 \mathrm{~S}}\right)>F_{\mathrm{AO}}>$ $F_{2 \mathrm{~A}}\left(F_{2 \mathrm{~S}}\right)$.

(3) The pneumatic circumferential force is the primary determinant of the vibration frequencies of the roundabout swing, the relationship being quadratic. The effect of the centrifugal force on the vibration frequencies is relatively weak.

\section{Competing Interests}

The authors declare that they have no competing interests.

\section{Acknowledgments}

This study was supported by the National Natural Science Foundation of China (Grant no. 51466012), the Inner Mongolia Natural Science Foundation of China (Grant no. 2016MS0509), and the Open Foundation of Key Laboratory of Wind Energy and Solar Energy Technology of the Ministry of Education of China (Grant no. 201408).

\section{References}

[1] D. A. Spera, Wind Turbine Technology, ASME Press, New York, NY, USA, 1995.

[2] H. Hamdi, C. Mrad, A. Hamdi, and R. Nasri, "Dynamic response of a horizontal axis wind turbine blade under aerodynamic, gravity and gyroscopic effects," Applied Acoustics, vol. 86, pp. 154-164, 2014.

[3] K. Bassett, R. Carriveau, and D. S.-K. Ting, "Vibration response of a $2.3 \mathrm{MW}$ wind turbine to yaw motion and shut down events," Wind Energy, vol. 14, no. 8, pp. 939-952, 2011.

[4] C. Van der Woude and S. Narasimhan, "A study on vibration isolation for wind turbine structures," Engineering Structures, vol. 60, pp. 223-234, 2014.

[5] K. McLaren, S. Tullis, and S. Ziada, "Measurement of high solidity vertical axis wind turbine aerodynamic loads under high vibration response conditions," Journal of Fluids and Structures, vol. 32, pp. 12-26, 2012.

[6] J. W. Wang, J. X. Yan, J. P. Liu, and Z. Zhao, "Experimental modal analysis of multi-blade wind turbine," Acta Energiae Solaris Sinica, vol. 29, no. 12, pp. 1460-1464, 2008.

[7] V. Maldonado, M. Boucher, R. Ostman, and M. Amitay, "Active vibration control of a wind turbine blade using synthetic jets," International Journal of Flow Control, vol. 1, no. 4, pp. 227-237, 2009.

[8] S.-M. Lin, S.-Y. Lee, and Y.-S. Lin, "Modeling and bending vibration of the blade of a horizontal-axis wind power turbine," Computer Modeling in Engineering and Sciences, vol. 23, no. 3, pp. 175-186, 2008.

[9] K. A. Brown and R. Brooks, "Design and analysis of vertical axis thermoplastic composite wind turbine blade," Plastics, Rubber and Composites, vol. 39, no. 3-5, pp. 111-121, 2010.

[10] M. V. Bastawrous and A. A. El-Badawy, "A study on coupled bending and torsional vibrations of wind turbine blades," Advanced Materials Research, vol. 622, pp. 1236-1242, 2013.

[11] K. Deines, T. Marinone, R. Schultz et al., "Modal analysis and SHM investigation of CX-100 wind turbine blade," in Rotating Machinery, Structural Health Monitoring, Shock and Vibration, vol. 5 of Conference Proceedings of the Society for Experimental Mechanics Series, pp. 413-438, Springer, 2011. 
[12] R. Osgood, G. Bir, H. Mutha et al., "Full-scale modal wind turbine tests: comparing shaker excitation with wind excitation," in Structural Dynamics and Renewable Energy, Volume 1: Proceedings of the 28th IMAC, A Conference on Structural Dynamics, 2010, Conference Proceedings of the Society for Experimental Mechanics Series, pp. 113-124, Springer, Berlin, Germany, 2011.

[13] D. T. Griffith and T. G. Carne, "Experimental modal analysis of 9-meter research-sized wind turbine blades," in Structural Dynamics and Renewable Energy, vol. 1 of Conference Proceedings of the Society for Experimental Mechanics Series, pp. 1-14, Springer, 2011.

[14] S. N. Ganeriwala, J. Yang, and M. Richardson, "Using modal analysis for detecting cracks in wind turbine blades," Sound and Vibration, vol. 45, no. 5, pp. 10-13, 2011.

[15] J. F. Kusnick and D. E. Adams, "Vertical axis wind turbine operational modal analysis in sheared wind flow," in Topics in Experimental Dynamics Substructuring and Wind Turbine Dynamics, Volume 2: Proceedings of the 30th IMAC, A Conference on Structural Dynamics, 2012, Conference Proceedings of the Society for Experimental Mechanics Series, pp. 333-344, Springer, Berlin, Germany, 2012.

[16] M. Garcia, A. Reich, and Y. J. Zhu, "Real-time dynamic measurements of a wind turbine rotor blade using modal filtering," in Topics in Model Verification and Validation, Volume 1: Proceedings of the 27th IMAC, A Conference and Exposition on Structural Dynamics, 2009, Conference Proceedings of the Society for Experimental Mechanics, pp. 214-217, Springer, Orlando, Fla, USA, 2009.

[17] T. G. Carne and A. R. Nord, Modal Testing of a Rotating Wind Turbine, The American Solar Energy Society, 1983.

[18] Y. Bazilevs, M.-C. Hsu, J. Kiendl, R. Wüchner, and K.-U. Bletzinger, "3D simulation of wind turbine rotors at full scale. Part II: fluid-structure interaction modeling with composite blades," International Journal for Numerical Methods in Fluids, vol. 65, no. 1-3, pp. 236-253, 2011.

[19] D. Macphee and A. Beyene, "Fluid-structure interaction of a morphing symmetrical wind turbine blade subjected to variable load," International Journal of Energy Research, vol. 37, no. 1, pp. 69-79, 2013.

[20] S. G. Horcas, F. Debrabandere, B. Tartinville et al., "Mesh deformation tool for offshore wind turbines fluid-structure interaction," in Proceedings of the 11th World Congress on Computational Mechanics, vol. 7, pp. 5139-5150, Barcelona, Spain, July 2014. 


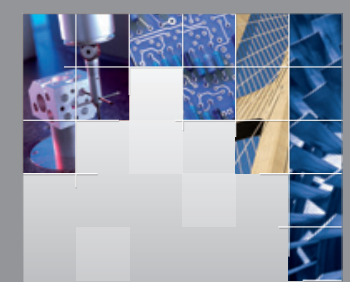

\section{Enfincering}
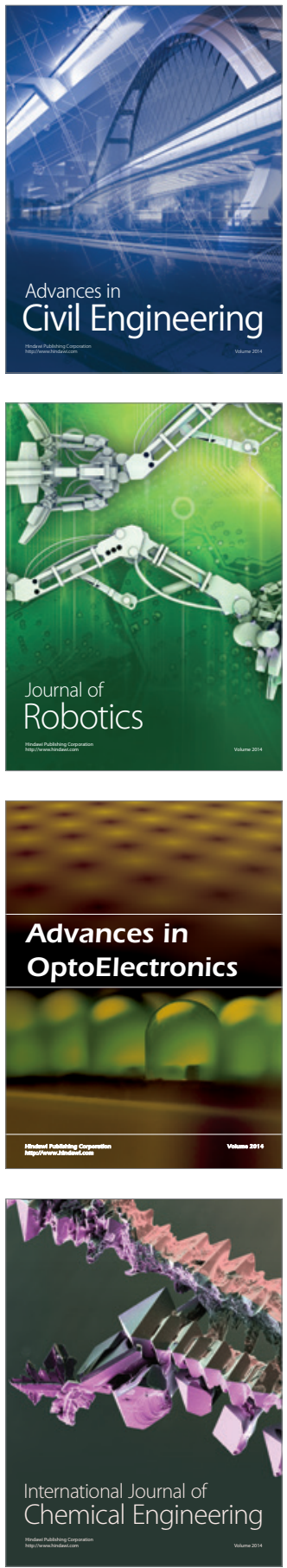

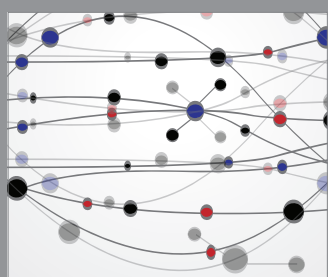

The Scientific World Journal

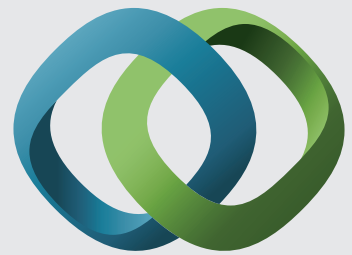

\section{Hindawi}

Submit your manuscripts at

http://www.hindawi.com
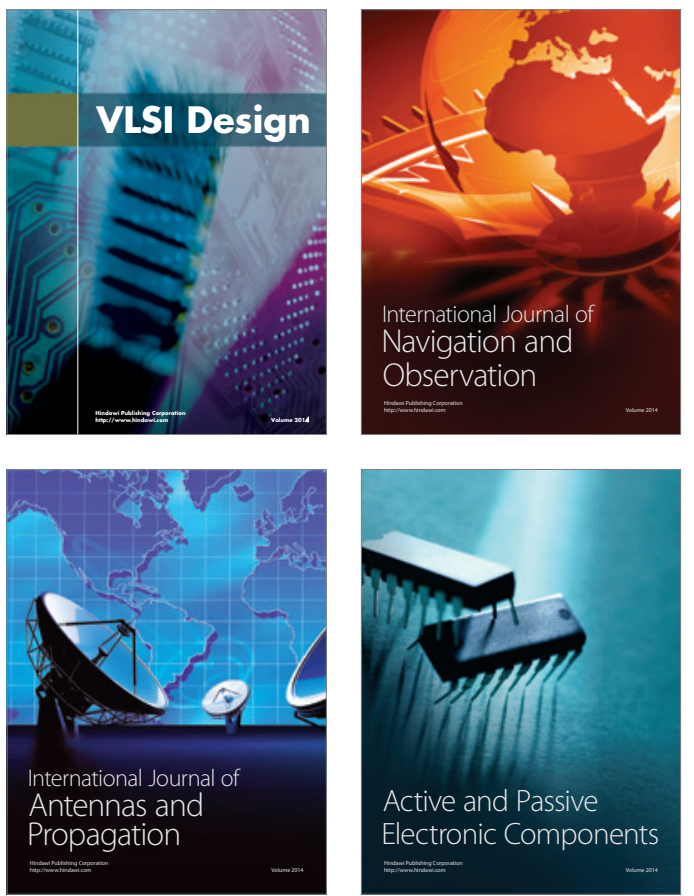
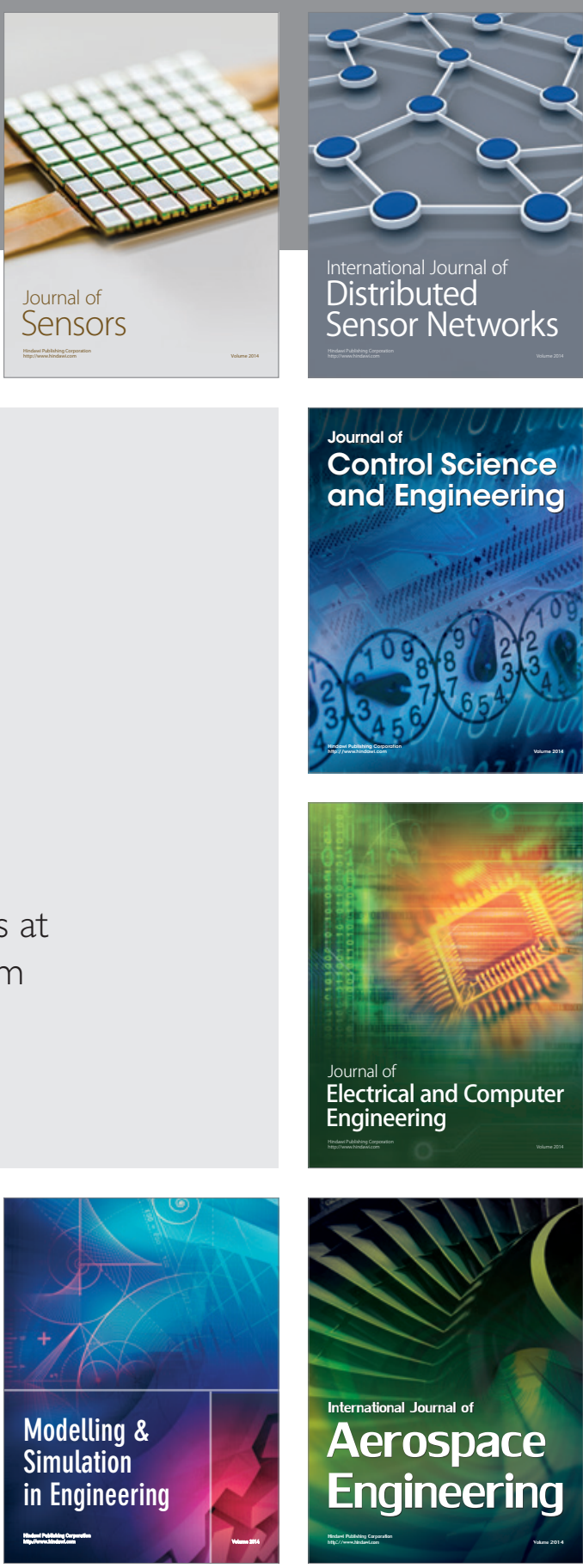

International Journal of

Distributed

Sensor Networks

Journal of

Control Science

and Engineering
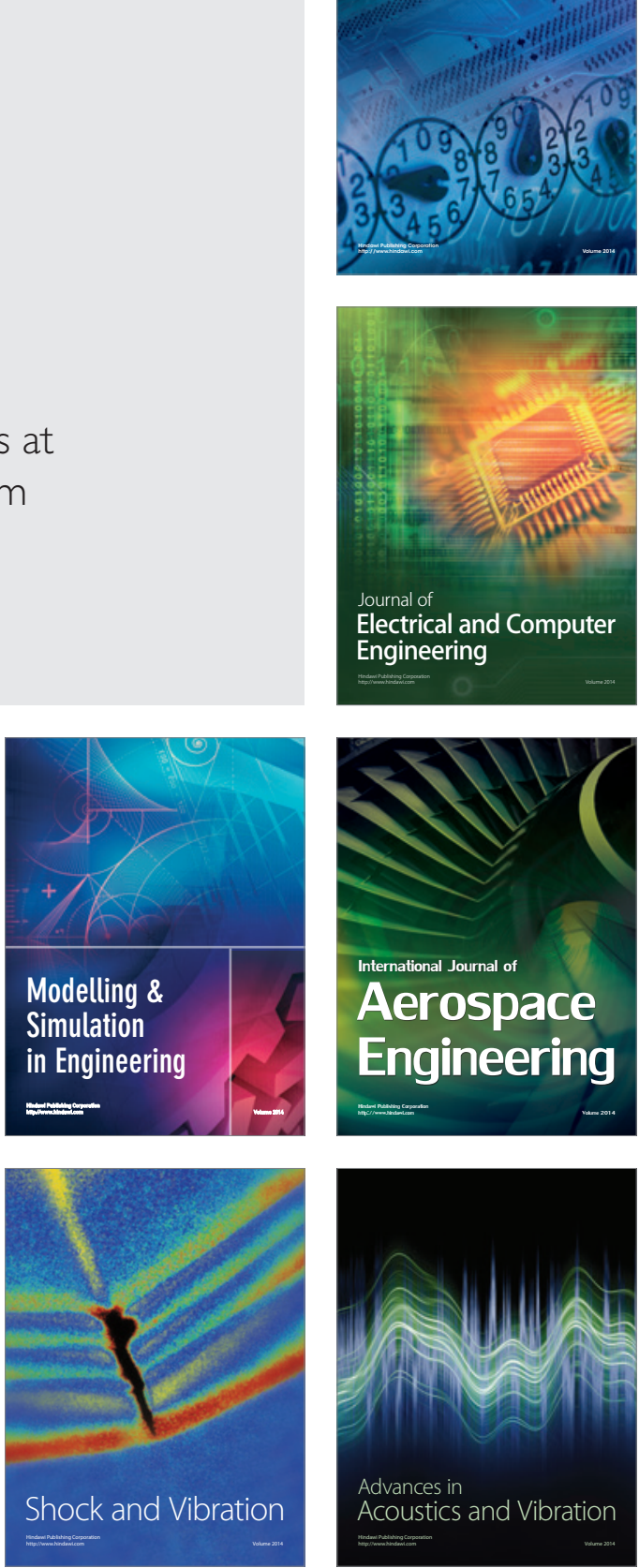